\title{
An Improved Segmentation Approach for Skin Lesion Classification
}

\author{
Youssef Filali ${ }^{1, *}$, My Abdelouahed Sabri ${ }^{1}$, Abdellah Aarab ${ }^{2}$ \\ ${ }^{1}$ LIIAN, Department of computer science,Faculty of Sciences Dhar-Mahraz, Sidi Mohamed Ben Abdellah University \\ ${ }^{2}$ LESSI, Department of physics,Faculty of Sciences Dhar-Mahraz, Sidi Mohamed Ben Abdellah University
}

\begin{abstract}
Skin cancer is considered as one of the dangerous types of cancer with a high proportion of deaths. This cancer can be categorized into two main types; Melanoma, which is the deadliest form, and Non-Melanoma. Early melanoma detection and diagnosis allows more treatment options and decreases significantly the number of deaths. Many researchers proposed to use image processing for skin lesion detection. The process can be divided into three main stages: lesion identification based on image segmentation, features extraction and lesion classification. Segmentation and features extraction are the key-steps and significantly influence the outcome of the classification results. In this paper, an improved segmentation approach for skin lesion detection and classification has been proposed. The proposed approach consists on a pre-processing based on multiscale decomposition thats separate the input image into two components. The geometrical component will be used in the segmentation stage and the texture component in features extraction. The efficiency and the performance of the proposed approach has been evaluated in comparison with recent and robust dermoscopic approaches from literature.
\end{abstract}

Keywords Skin cancer, PDE Multi-scale decomposition, Texture analysis, Features extraction, SVM.

AMS 2010 subject classifications 68T05, 68T10, 68T45

DOI: $10.19139 /$ soic.v7i2.533

\section{Introduction}

Skin lesion analysis is considered a wide study area. It plays a great role in skin cancer prevention, specifically in terms of getting an effective early diagnosis. These lesions can be classified mainly as malignant (melanoma) or benign (not-melanoma). A growing number of deaths due to melanoma has been noticed globally, this type of malignant in Figure. 1 is deemed to be the most aggressive one compared to other lesion. Not melanoma lesions show a very organized structure than melanoma lesions.

Due to the visual aspect of the skin lesions, various non-invasive imaging approaches have been used to help dermatologist in detecting and classifying skin lesions. Macroscopic images, commonly known as clinical images, are basically used in computational analysis of skin lesions. However, their imaging conditions are frequently irregular and may have weak resolution, which is probably challenging when the images under study are tiny. Another problem with clinical images is associated to the presence of artefacts, reflections, like hair, and skin lines that might thwart adequate analysis of the imaged skin lesions.

Many researchers proposed to use image processing for skin lesion detection. The process can be divided into three steps: lesion identification based on image segmentation, features extraction and lesion classification. Segmentation and features extraction are the key-steps and significantly influence the outcome of the classification results. The aim of segmentation is to identify and extract from the macroscopic image under analysis the lesion area. Generally,

*Correspondence to: Youssef Filali (Email: youssef.filali1@usmba.ac.ma).

ISSN 2310-5070 (online) ISSN 2311-004X (print)

Copyright (C) 2019 International Academic Press 


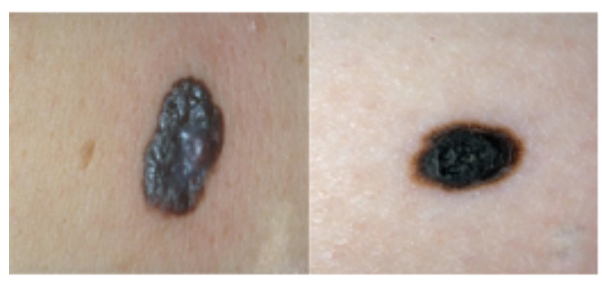

Figure 1. Examples of skin lesions.

skin images contain many type of noises and their segmentation is a very hard task and need some preprocessing. Several approaches based on color space transformation, contrast enhancement and artefact removal have been suggested to enhance the segmentation step.

Features extraction is usually based on the rules used by dermatologists in their clinical routine diagnosis. They use the ABCD rules as a visual classification criteria based on the Asymmetry, Border, Color and Diameter characteristics of the lesion under research $[1,2]$. An asymmetric lesion is automatically classified as malignant. The border criterion matches the lesions form measurement; normal form is associated to benign lesions while an irregular border is associated with malignant lesion. The color criterion consists of analyzing the tonality variation of the pigmented skin lesion to define the malignant lesions that normally present non-uniform colors. Texture analysis may also be used for image-based examination of skin lesion, since it helps in discriminating benign from malignant lesions by valuing the harshness of their structure. Lesion classification, based on the features extracted is the last step.

To help dermatologists in making their diagnosis, the aim of this work is to propose an improved skin lesion analysis and classification approach based on texture, asymmetry and color features. A multiscale decomposition preprocessing is used to reduce first the noise present in the image and to isolate the textures form object (lesion). In the segmentation step of the lesion in the preprocessed image, any efficient segmentation algorithm can be applied. In this work, the K-means is adopted as segmentation algorithm. Three categories of features are used: (i) textural features from texture component obtained after decomposition: Gray Level Co-Occurrence Matrix (GLCM), Gabor filter, Histogram of Oriented Gradient (HOG) and Local Binary Pattern (LBP), (ii) skin lesion asymmetry measurement from the object component, and (iii) color features of the lesion from the original image. Finally, to classify the skin lesion the features are introduced as an input matrix to the Support Vector Machine (SVM) classifier.

The rest of the paper is organized as follows; in Section I a review related works of skin lesion classification is presented. Section II gives a description of the proposed approach. The given results will be discussed in Section III. In Section IV evaluation of the proposed approach in presented, followed by a conclusion.

\section{Related studies}

Computer Aided Diagnosis (CAD) systems are used for an early skin cancer diagnosis. It generally consists of three steps: segmentation, features extraction, and classification. A preprocessing step is always performed to increase the segmentation results. A Median filter is applied to smooth and remove artifacts. Also, it preserves the border and keeps pertinent information of the lesion [3, 4]. Anisotropic Diffusion filter is better used to smooth the skin lesion and remove artifacts to get better results. A Morphological filter helps remove noise and may also be applied to improve segmentation and to encompass an area with a more regular border [5].

In image segmentation, many techniques are used to help in the identification of the skin lesion. Thresholding algorithms have been used on a large scale due to their simplicity. Otsu is the most used algorithm in automatic thresholding segmentation [6,7]. Algorithms based on active contour models have been continuously suggested for segmentation of skin lesion in images where the initial curves are being moved to the object of interest through convenient deformation [7, 8, 9]. Region based algorithms have also been widely used to segment skin 
lesion images. For example, regions growing, spliting, and merging. There are many algorithms based on artificial intelligence[10], which are justified by the advantages that they offer. One advantage that they offer is the possibility to learn from the sample case provided by the artificial neural networks [11]or the deep neuronal network [12]. Other segmentation algorithms arise from the search and optimization, such as the genetic algorithms. In addition, Fuzzy logic [13]joined with clustering techniques have been applied in image segmentation of skin lesion such as the Fuzzy C-Means(FCM) and K-means algorithms [14, 15]. Yet, some disadvantages might occur by algorithms based on Artifical Intelligence concerning the complexity of the implementation and the presence of many steps, which demands high computational efforts.

In the features extraction step, based on the identification of the regions of interest (ROIs) in the image, authors in [1] proposed to use rules used by dermatologists. ABCD (Asymmetry, Border, Color and Diameter) characteristics and the GLCM measeures are the most used[16, 17]. For the process of classification, more than one method has been evaluated to achieve the best outcome $[18,19]$. The performance depends on several issues like the quality of the segmentation images, the extracted features, and the classification algorithms. Therefore, the output may either be binary class or multi-class $[18,20]$.

\section{THE PROPOSED APPROACH}

Segmentation has a major influence on the quality of the features and thus on the result of the classification results. In this part, a multi-scale approach is presented to classify the pigmented lesion and provide an approach that help dermatologists in their diagnosis. Figure. 2 explains this given approach, which involves the following steps:

1. Image preprocessing: Each RGB canal of the input image will be decomposed separately into texture and object components using the adopted multi-scale decomposition,

2. Image segmentation: The segmentation is performed only on the object components. The extracted ROI will be used to identify its paired region from the texture component,

3. Features extraction: The features are extracted separately from the two components. Textural features will be extracted from texture component, asymmetry measure is extracted from the object component, and color features are extracted from the lesion in the original image,

4. Classification.

\subsection{Multi-scale decomposition models}

The presence of the texture in skin cancer images is the major problem encountered for segmentation and consequently for lesion classification. Bad segmentation involves incorrect identification of the lesion and will negatively influence its classification results. The idea proposed is to use a multi-scale decomposition model to separately extract the texture and the object into components and then to process each of them individually. The segmentation is performed only on the object component and not the texture component. Instead, the textural features will be extracted from the texture component. The preprocessing based on the multi-scale decomposition is able to remove the artifacts (contrast, hairs, blood vessels, skin lines) and facilitate the border detection.

In order to make perplexed image and vision problems resolved, Partial Differential Equations (PDE) can be used. The most common PDE is the ROF model. The objective is to separate the image into two components $u$ and $\mathrm{v}$ where the first component, $\mathrm{u}$, is well structured and has the whole geometric information of the image and the second component, $v$, contains oscillating information such as textures and possible noise [21].

The Aujol decomposition model used is presented by the equation (1) [22].

$$
F_{(\lambda, \mu)}(\mu, v, w)=J(\mu)+J \times\left(\frac{v}{\mu_{1}}\right)+J \times\left(\frac{w}{\mu_{2}}\right)+\frac{1}{2 \lambda}\left\|f-\mu-v_{1} v-v_{2} w\right\|_{L_{2}}^{2}
$$




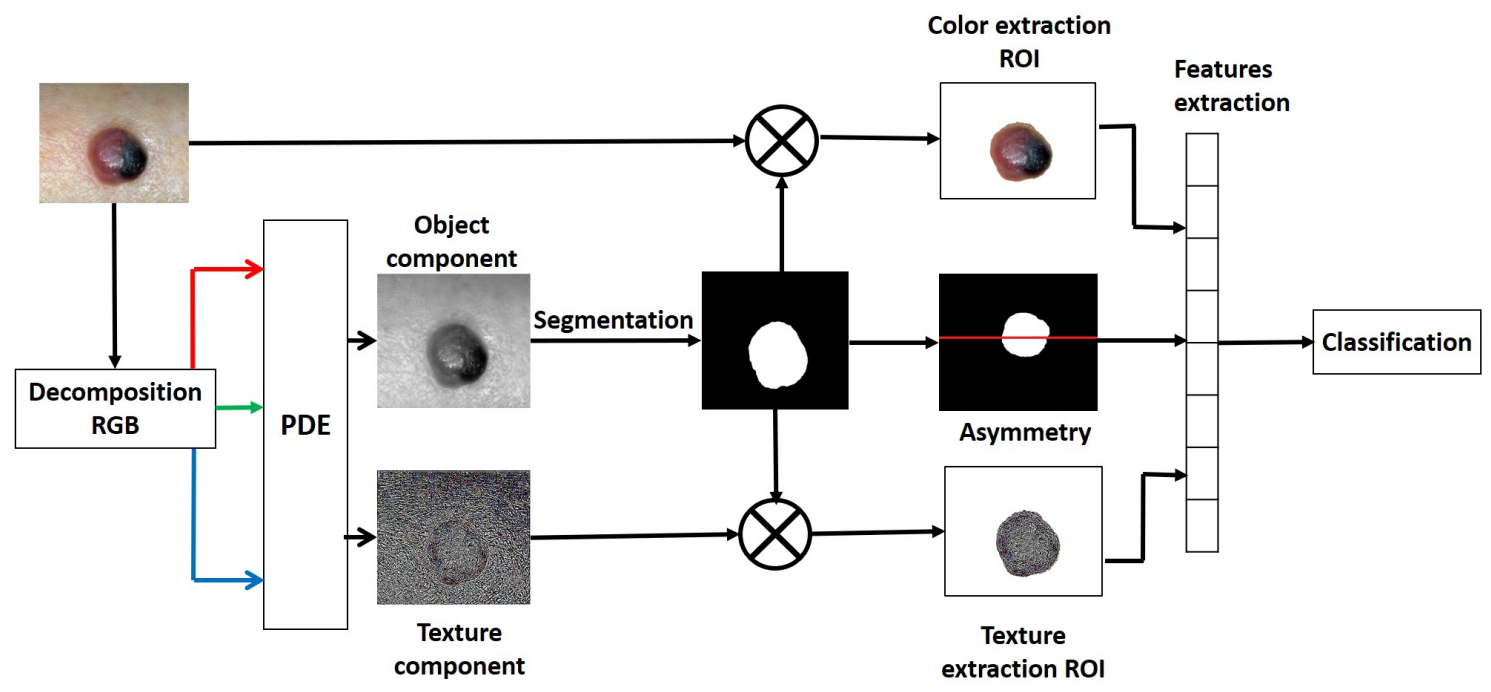

Figure 2. The workflow of the proposed approach.

This decomposition is given by minimizing a functional $\mathrm{f}$ where $v_{1}$ and $v_{2}$ are two functions in $\left.\left.R^{2} \longrightarrow\right] 0 ; 1\right]$ generalizing the idea of adaptive regularization coefficient according to the image area in which there is. The functions $\mathrm{v}$ and $\mu$ can be seen both as oscillators, taken respectively in $G \mu_{1}$ and $G \mu_{2}$. The function u will be taken in the BV space.

\subsection{Segmentation}

Since the segmentation is performed on the object component which not contains any texture, a simplest segmentation algorithm can gives a good results. The K-means algorithm is adopted here based on our previous works $[14,23]$. K-Means is appropriate for skin image segmentation, especially for lesion detection, as the number of clusters $\mathrm{k}$ is usually known [14].

\subsection{Features extraction}

Features extraction are the key of a successful classification system. It consists in extracting pertinent information, from skin lesion image, able to describe effectively the lesion and used to classify the lesion as a melanoma or not. The features used in literature are especially based on the ABCD (Asymmetry, Border, Color and Diameter) rules. Features used in this paper to classify the lesions are textural, color and the Asymmetry criteria.

\subsubsection{Textural features}

\section{- Gray Level Co-Occurrence Matrix (GLCM)}

GLCM is used in our work in order to offer better extraction of textural features from the skin lesion images. It characterize the spatial distribution and the grey levels inside a restricted surface. Under a predetermined angle and distance, the $(\mathrm{I}, \mathrm{j})$ entries of every matrix offer the possibility for it to be accepted from one pixel with a grey level of $i$ to another level $\mathrm{j}$. Feature vectors, which are the result of these settings, are now considered and the matrix is created from both the orientation and the distance between the pixels of the image.

From [21], we note that Haralick offers fourteen different textural features, which were the result of calculating the probability matrix that extracts the characteristics of texture statistics from the remote sensing 
images. However, each essential statistic is bound to a different texture type. Energy, Contrast, Correlation, Homogeneity, and Entropy are the statistics used:

Energy: measures the texture disorder

$$
E=\sum x, y p(x, y)^{2}
$$

$\mathrm{P}(\mathrm{x}, \mathrm{y})$ the normalized and symmetrical GLCM

Contrast: the key diagonal close the moment of inactivity, which amount the importance of the matrix is dispersed and images of limited variations in number, reflecting the image clearness and texture of shadow depth.

$$
I=\sum \sum(x-y)^{2} p(x, y)
$$

Entropy: produces a measure of randomness, having its highest value when the all elements equal.

$$
S=-\sum x, y p(x, y) \log p(x, y)
$$

Correlation Coefficient: Procedures the joint probability incidence of the identified pixel pairs.

$$
C=\sum\left(\sum((x-\mu x)(y-\mu y) p(x, y) / \sigma x \sigma y)\right)
$$

Homogeneity: Methods the nearness of the scattering of elements in the GLCM to the GLCM diagonal.

$$
H=\sum\left(\sum(p(x, y) /(1+[x-y]))\right)
$$

\section{- Gabor filter}

Gabor filter have been applied in different and many areas of image processing such as edge detection, texture classification, fingerprint identification [24, 25]. Gabor wavelets are used to extract texture features from images [26]; the idea is based on detecting linear directional elements.

Gabor filters decomposes the skin lesion image into components corresponding to different scales and different orientations:

$$
h(x, y)=\frac{1}{2 \Pi \sigma_{x} \sigma_{y}} \exp \left\{-0.5\left[\frac{x^{2}}{\left(\sigma_{x}^{2}\right)}+\frac{y^{2}}{\left(\sigma_{y}^{2}\right)}\right]\right\} \exp (j 2 \Pi F x)
$$

Where $\mathrm{F}$ denotes the radial frequency of the Gabor function. The space constants $\sigma_{x}$ and $\sigma_{y}$ define the Gaussian envelope along the $\mathrm{x}$ - and $\mathrm{y}$ - axes. The Gabor filter with frequency $\mathrm{F}$ and orientation $\theta$ by coordinate rotation given by:

$$
h^{\prime}(x, y)=\frac{1}{2 \Pi \sigma_{x} \sigma_{y}} \exp \left\{-0.5\left[\frac{x^{\prime 2}}{\left(\sigma_{x}^{2}\right)}+\frac{y^{\prime 2}}{\left(\sigma_{y}^{2}\right)}\right]\right\} \exp \left(j 2 \Pi F x^{\prime}\right)
$$

\section{- Histogram of Oriented Gradient (HOG)}

HOG is a descriptor used in computer vision and image processing in object detection and features extraction. It counts gradient orientation occurrences in restricted portions of an image.

The idea of the histogram of oriented gradients descriptor is that local object appearance and shape within an image can be described by the distribution of intensity gradients or edge directions. The descriptor is the concatenation of all the compiled histograms of oriented gradients for each connected region (cells) in the image. All the histograms are constrast-normlized by measuring the intensity accros the region to improve the accuracy. the advantage of this descriptor remains in the fact that it is invariant to geometric and illumintaion transformation.

\section{- Local Binary Pattern (LBP)}


3.3.2. Color features: In order to calculate the color that the lesion contains, according the literature the melanoma are described by the presence of six different colors that are, white, red, light brown, dark brown, blue-gray and black.

$$
\begin{gathered}
\text { Black } \left.=\sum(R<0.2 \& G<0.2 \& B<0.2)>0\right) \times 100 / N B \\
\left.\operatorname{Red}=\sum(R<0.8 \& G<0.2 \& B<0.2)>0\right) \times 100 / N B \\
\text { Bluegray } \left.=\sum(R<0.2 \& G>0.32 \& G<0.72 \& B>0.34 \& B<0.74)>0\right) \times 100 / N B \\
\text { White }=\sum((R>0.8 \& G>0.8 \& B>0.8)>0) \times 100 / N B \\
\text { Lightbrown } \left.=\sum(R>0.6 \& R<1 \& G>0.32 \& G<0.72 \& B>0.05 \& B<0.45)>0\right) \times 100 / N B \\
\text { Darkbrown } \left.=\sum(R>0.2 \& R<0.6 \& G>0.06 \& G<0.46 \& B>0 \& B<0.33)>0\right) \times 100 / N B
\end{gathered}
$$

with NB is the number of pixels in the lesion. R, G and B are consecutively the red, green and blue components in the RGB representation. The six feature is obtained by adding the colors.

3.3.3. Asymmetry feature The asymmetry criterion can be tested by separating the regions of the lesion into two sub-regions by an axis of symmetry. In order to analyze the correspondence of the area, the two sub-regions must be folded over each other along the axis. If there is any part of either sub-region not overlapped, then the lesion is considered asymmetrical. Asymmetry in a lesion is the most important indicator of malignancy.

The axis of symmetry, otherwise known as the major axis, is found by identifying the center of the lesion, the two foci, and the longest diameter. The line must cross the center and the two foci and it must end at the widest points of the perimeter in order to be identified as the major axis. Finally, we rotate the major axis to be parallel to the horizontal axis.

\subsection{Classification}

The next phase is to use the set of features extracted to classify. The dataset is first divided randomly into two sets; training and test set. The training set consists of developing a classification model based on the training samples, which are the input data to the classifier for the learning process and the test set is to evaluate the classification accuracy.

Several algorithms can be used for classification and accuracy is basically based on the features relevance. In this work, the choice of the classification tool in not a big deal and will not influence significantly on the classification results. The Support Vector Machine (SVM) [23] is the most used classifier in literature for lesion skin classification.

Classification with SVM is an example of Supervised Learning, it looks for finding the optimal separating hyper plane between classes, by opting the hyper plane with the maximal margin. To verify whether the system is performing in a right way or not and to validate the accuracy of the proposed system, the predicted labels will be compared to the already known ones is shown the help of the known labels. 


\section{Experimentation}

In this part, the given results of segmentation and classification is presented and discussed. The images-set used to evaluate the proposed approach is Atlas Dermoscopy [27]. It contains 80 images of pigmented skin lesion manually segmented into Ground of Truth (GT) and classified into melanoma and not melanoma by dermatologists. In Figure. 3 and Figure. 4 an example of images form database is presented:

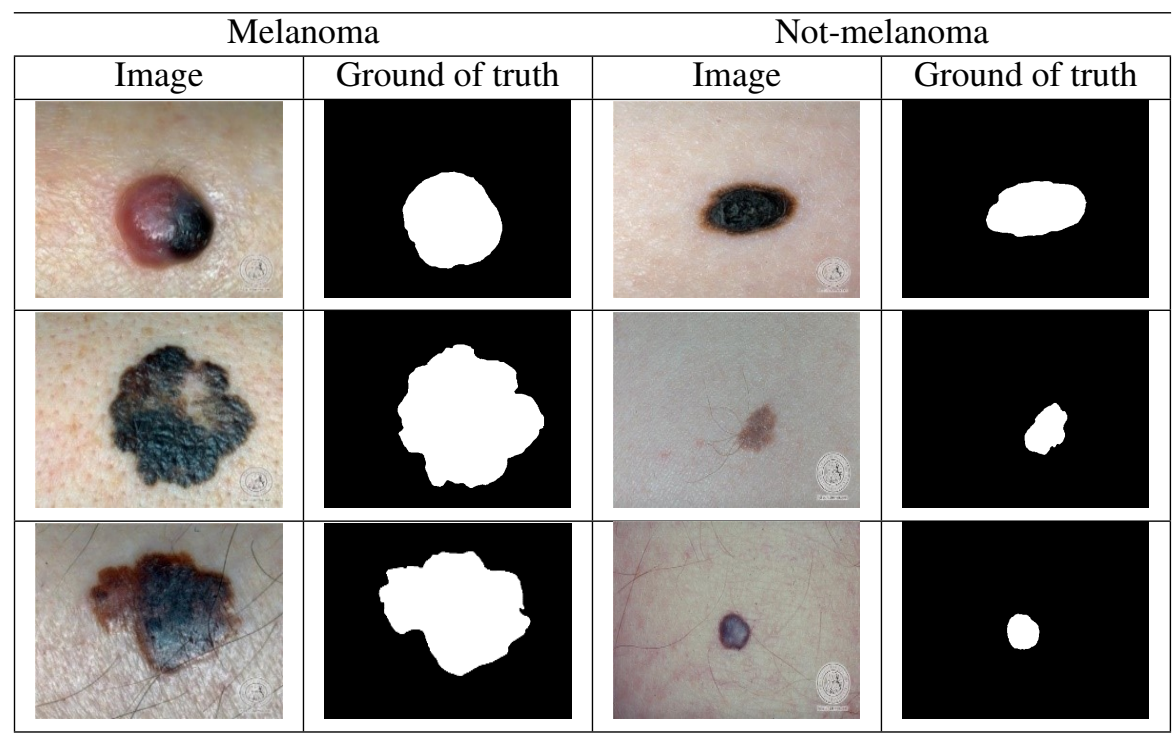

Figure 3. Example of images from Atlas Dermoscopy dataset

The experimentation will be conducted in three steps:

1. Segmentation evaluation using our proposed preprocessing decomposition in comparison with the segmented GT.

2. Features extraction comparison between Gray Level Co-Occurrence Matrix (GLCM) and Gabor filter Local Binary Pattern (LBP) and Histogram of Oriented Gradient (HOG), and then concatenated with features of color and the Asymmetry that contain the lesion.

3. Lesion classification accuracy of the proposed approach in comparison with known approaches form literature.

\subsection{Preprocessing}

In order to remove noise and artefact form the lesions, the decomposition using Aujol Model is conducted on each of the RGB canals. Therefore, every canal is decomposed separately into two components; texture and object. The texture and object components of the input image is the concatenation of the three components for each canal.

The segmentation is performed on only the object component that contains only objects shape without any texture or noise. Sensitivity (TP rate) and Specificity (TN rate) measures are used to evaluate the segmentation results in regards on the GT.

$$
\begin{aligned}
& \text { Sensitivity }=\frac{T P}{(T P+F N)} \\
& \text { Specificity }=\frac{T N}{(F P+T N)}
\end{aligned}
$$


Where: TP is the number of true positives,

$\mathrm{TN}$ is the number of true negatives,

FP is the number of false positives

FN is the number of false negatives

Figure. 4 presents respectively the segmentation results of an image selected randomly from the database without and with pre-processing by the Aujol model. It also presents the colored skin lesion and the textured skin lesion after projection of the segmented image on respectively original image and texture component.

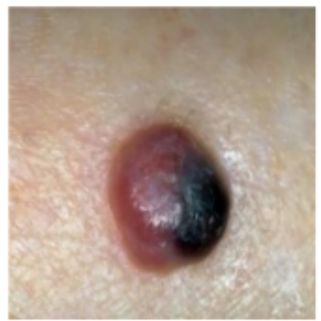

Original image

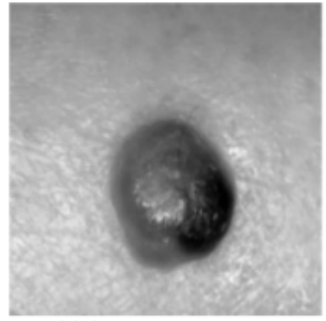

Object component

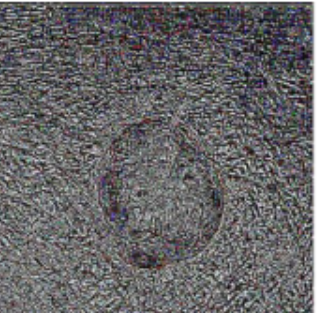

Texture component

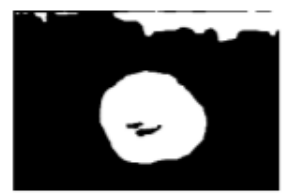

Segmentation without preprocessing

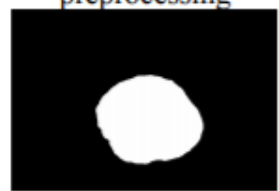

Segmentation of the Object component

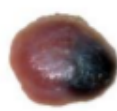

Color skin lesion

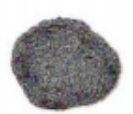

Texture skin lesion

Figure 4. Segmentation result using with and without preprocessing via the Aujol decomposition

For the segmentation evaluation, Table 1 presents the result of the Sensitivity and Specificity of the proposed approach. The ground truth (GT) for each case-study is used to estimate the TP, TN, FP and FN used to calculate the Sensitivity and Specificity.

Table 1. EVALUATION OF THE SEGMENTATION RESULT IN TERM OF SENSITIVITY AND SPECIFICITY

\begin{tabular}{|c|c|c|}
\hline & Without Aujol decomposition & With Aujol decomposition \\
\hline Sensitivity & 83.33 & 93.00 \\
\hline Specificity & 80.00 & 95.00 \\
\hline
\end{tabular}

From the Figure 4 and Table 2 it is clear that the segmentation of the object component is very promising and can effectively identify the skin lesion. In our first experimentation we use the K-Means in segmentation knowing that there are in the literature other segmentation algorithms. Thereafter another comparison is conducted to evaluate 
the best segmentation approach to use and to justify the use of the K-Means. In table reftable2 we present the sensitivity and the specificity of the segmentation using K-Means, Otsu, FCM, Region merge and ExpectationMaximization [3, 7, 10, 26, 28].

Table 2. AVERAGE RESULTS USING DIFFERENT SEGMENTATION ALGORITHMS IN TERM OF SENSITIVITY AND SPECIFICITY

\begin{tabular}{|l|c|c|c|c|c|}
\hline & Expectation-Maximization & Otsu & Fuzzy C-means & Region merge & K-Means \\
\hline Sensitivity & 90.00 & 93.33 & 90.00 & 93.33 & 96.67 \\
\hline Specificity & 85.00 & 95.00 & 90.00 & 90.00 & 95.00 \\
\hline
\end{tabular}

From results in Figure 4, Table 1 and Table 2 we can conclude that the use of the Aujol decomposition as a preprocessing step improve significantly the segmentation result and the choice of k-Means is justified.

\subsection{Classification}

Features extraction is the key of the success of a classification method. The issue with lesion image classification is that the segmentation does not identify correctly the lesion region and the features extracted are not very pertinent. The decomposition of the original image by Aujol model gives us the opportunity to segment only the objects without textures. That is why the performance of the segmentation presented in the last section was very convincing. Once we have the segmented region, we will project it on the texture component to create powerful features. The features used will be based on textural, asymmetry and color information extracted from the lesion. The Gray Level Co-Occurrence Matrix (GLCM), Gabor filter, Histogram of Oriented Gradient (HOG) and Local Binary Pattern (LBP) was presented as most used for textural features extraction. As we say before, the most important task is the extraction of features and the SVM will be used to evaluate the classification rate. The classification measure used in this paper is the Accuracy

$$
\text { Accuracy }=\frac{(T P+T N)}{(T P+T N+F P+F N)}
$$

A first study conducted to select the best features obtained for different scale and orientation for Gabor filter. In Figure 5 we present the classification accuracy using Gabor features for different number of scales and directions and we can conclude that the best features can be obtained for a scale and orientation values equals to 3 and 8 respectively.

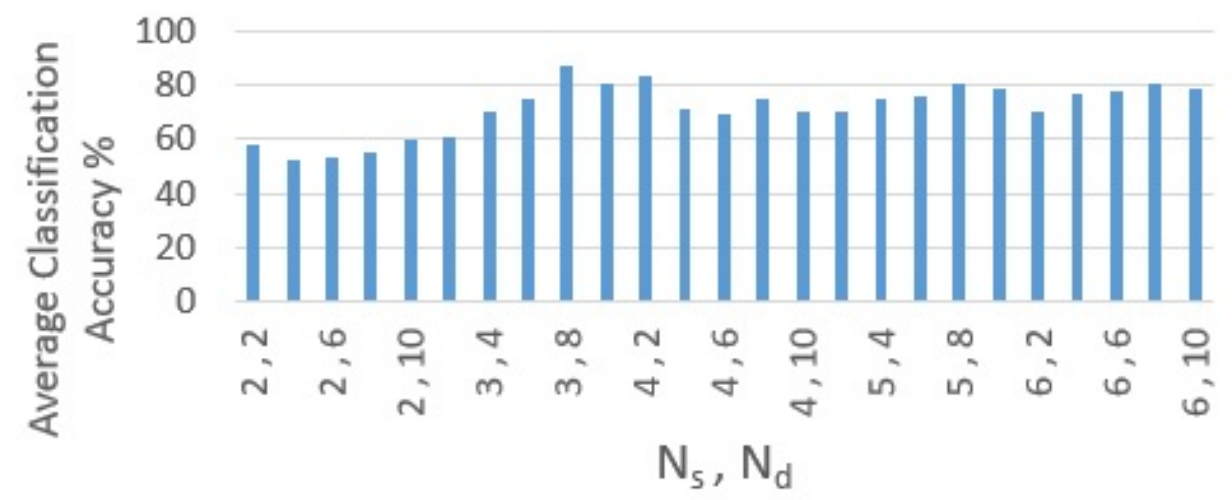

Figure 5. SVM Classification accuracy, using Gabor features, for different number of scales and directions

For the GLCM features, we extract 13 features, for Gabor filter we extract 160 features, LBP 59 features and for 
the HOG 34596 features are extracted. The classification accuracy for the different features extracted separately is presented in Table 3.

Table 3. SVM CLASSIFICATION ACCURACY USING DIFFERENT FEATURES EXTRACTION

\begin{tabular}{|l|c|c|c|c|}
\hline & Gray Level Co-Occurrence Matrix & Gabor filter & Histogram of Oriented Gradient & Local Binary Pattern \\
\hline Accuracy & 80.00 & 83.00 & 80.23 & 81.23 \\
\hline
\end{tabular}

The best accuracy is got by the Gabor filter $(83.00 \%)$, but remains not very satisfactory. In the next step, we will use the color features extracted from the lesion in the original image and the asymmetry of the lesion, then we compare the result with other approaches from literature.

In order to identify asymmetry of a lesion, we take the major axis and we rotate the image so this axis is parallel to the horizontal axis as presented by the red line in Figure 5. Then, we separate the two sub-regions and we fold them along this axis. If the two sub-regions are aligned perfectly then they considered are symmetric, whereas if there is some or a lot of overlap then the lesion is considered asymmetric. In our approach, we have a feature that determines the excess number of pixels that are not overlapped in asymmetrical lesions.

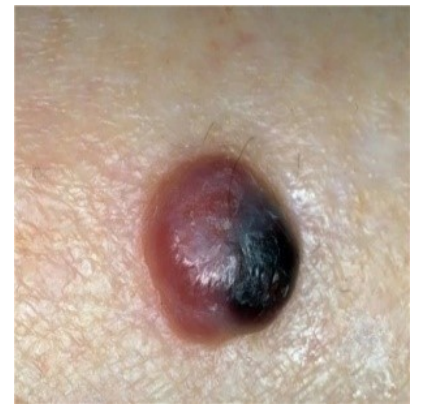

(a) Original image

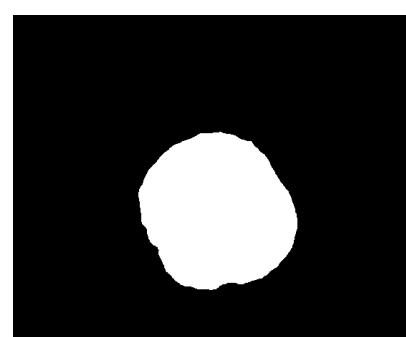

(b) Segmentation of the Object component

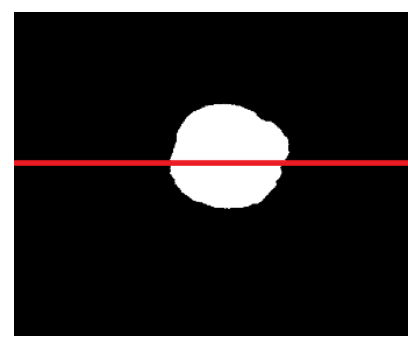

(c) Axis of symetry

Figure 6. Segmented lesion symmetry axis

As for the colors, we have a feature that contains values of the six possible colors and another feature that states which of the six are present. We combine the color feature with the asymmetry feature with the texture feature.

Table 4 presents a comparison between our proposed approach and four other approaches from literature.

R.Amelard proposes to use the High-Level Intuitive Features (HLIF) designed to model the ABCD criteria [29]. In his model each HLIF represents a human-observable characteristic. Alin M. Solomon uses the GLCM and color as a features extraction [30]. Almansour uses color features and texture features based on LBP in addition to GLCM [31]. In contrast, B. Gohila[32] uses only GLCM as a features extraction and PNN in classification. 
Table 4. SVM CLASSIFICATION ACCURACY OF THE PROPOSED APPROACH IN COMPARISON WITH OTHER APPROACHES FROM LITTERATURE

\begin{tabular}{|l|c|c|c|c|c|}
\hline & R.Amelard [29] & Alin M. Solomon [30] & Ebtihal Almansour [31] & B.Gohila vani [32] & Proposed approach \\
\hline Accuracy & 81.17 & 90.00 & 90.32 & 98.70 & 98.80 \\
\hline
\end{tabular}

From Table 4, we can clearly see that the accuracy with $98.80 \%$ of our proposed is higher than the other approaches. This is due, first, to the use of the Aujol decomposition which help us in the segmentation process. Also, the features used; Gabor filter, color, and asymmetry are very efficiency in describing the lesion.

\section{Conclusion}

Many solutions in image processing and analysis have been proposed for skin lesion to help dermatologists in their diagnosis. Knowing that the quality of the segmentation is, our main contribution is to improve the segmentation process to increase the accuracy of pigmented skin lesion classification. This approach is based on the Aujol decomposition model for structure and texture identification, k-means for segmentation, Gabor filter, asymmetry and color for features extraction and SVM as a classifier. The proposed approach was implemented and tested on medical images and achieved good segmentation results as well as good classification accuracy in comparison with other recent approaches from literature. As future work, concerning the segmentation and classification of skin lesion, we intend to use new algorithms and methods to develop more our systems of a higher quality diagnosis on dermatology images.

\section{REFERENCES}

1. D. N. L. Correa, L. R. B. Paniagua, J. L. V. Noguera, D. P. Pinto-Roa and L. A. S. Toledo, Computerized Diagnosis of Melanocytic Lesions Based on the ABCD Method, 2015 Latin American Computing Conference (CLEI), Arequipa, 2015, pp. 1-12.

2. S. Jain, V. Jagtap, and N. Pise, Computer aided melanoma skin cancer detection using image processing, Procedia Comput. Sci., International Conference on Computer, Communication and Convergence (ICCC 2015), vol. 48, no. C, pp. 736C741, 2015.

3. K. Norton, H. Iyatomi, M. E. Celebi, G. Schaefer, M. Tanaka, and K. Ogawa, Development of a Novel Border Detection Method for Melanocytic and Non-Melanocytic Dermoscopy Images, Annual International Conference of the IEEE Engineering in Medicine and Biology Society. IEEE Engineering in Medicine and Biology Society pp. 5403C5406, 2010.

4. Abbas and Qaisar et al., NA perceptually oriented method for contrast enhancement and segmentation of dermoscopy images, Skin research and technology : official journal of International Society for Bioengineering and the Skin (ISBS) [and] International Society for Digital Imaging of Skin (ISDIS) [and] International Society for Skin Imaging 191 (2013): e490-7.

5. Celebi ME, Aslandogan YA, Stoecker WV, Iyatomi H, Oka H, Chen X., Unsupervised border detection in dermoscopy images. Skin research and technology, official journal of International Society for Bioengineering and the Skin (ISBS) [and] International Society for Digital Imaging of Skin (ISDIS) [and] International Society for Skin Imaging (ISSI). 2007;13(4):454-462. doi:10.1111/j.16000846.2007.00251.x.

6. R. Garnavi, M. Aldeen, M. E. Celebi, A. Bhuiyan, C. Dolianitis, and G. Varigos, Automatic Segmentation of Dermoscopy Images Using Histogram Thresholding on Optimal Color Channels, Int. J. Med. Med. Sci., pp. 126C134, 2010.

7. R. Garnavi, M. Aldeen, M. E. Celebi, G. Varigos, S. Finch, A. Bhuiyan, and C. Dolianitis, Automatic segmentation of dermoscopy images using histogram thresholding on optimal color channels, Conputerized Med. Imaging Graph., vol. 35, no. January, pp. $105 \mathrm{C} 115,2011$.

8. A. Chopra and B. R. Dandu, Image Segmentation Using Active Contour Model, Int. J. Comput. Eng. Res., vol. 2, no. 3, pp. 819C822, 2012.

9. H. Zhou, G. Schaefer, M. Celebi, H. Iyatomi, K.-A. Norton, T. Liu, and F. Lin, Skin lesion segmentation using an improved snake model, JConf. Proc. IEEE Eng. Med. Biol. Soc., vol. 2010, no. 1, pp. 1974C7, 2010.

10. S. N. Deepa and B. Aruna Devi, A survey on artificial intelligence approaches for medical image classification, Indian J. Sci. Technol., vol. 4, no. 11, pp. 1583C1595, 2011.

11. S. Mathew and D. Sathyakala, Segmentation of skin lesions and classification by neural network, International Journal of Advanced Research in Electronics and Communication Engineering (IJARECE) Volume 4, Issue 2, February 2015.

12. J. Ko, S. M. Swetter, H. M. Blau, A. Esteva, B. Kuprel, R. A. Novoa, and S. Thrun, with deep neural networks, Nature, pp. 1C11, 2017.

13. A. A. I. Mohamed, M. M. Ali, K. Nusrat, J. Rahebi, and A. Sayiner, Melanoma Skin Cancer Segmentation with Image Region Growing Based on Fuzzy Clustering Mean, International Journal of Engineering Innovations and Research, vol. 6, no. 2, pp. 91C95, 2017. 
14. M. A. Sabri, A. Ennouni, and A. Aarab, Automatic estimation of clusters number for K-means, 2016 4th IEEE International Colloquium on Information Science and Technology (CiSt). Pages: 450 - 454, DOI: 10.1109/CIST.2016.7805089. Electronic ISSN: 2327-1884. 24-26 Oct. 2016.

15. M. A. Sabri; A. Ennouni; A. Aarab, Robust approach for textured image clustering, 2016 4th IEEE International Colloquium on Information Science and Technology (CiSt). Pages: 465 - 470, DOI: 10.1109/CIST.2016.7805089. Electronic ISSN: 2327-1884. 24-26 Oct. 2016. Tangier, Morocco

16. P. Mohanaiah, P. Sathyanarayana, and L. Gurukumar, Image Texture Feature Extraction Using GLCM Approach, International Journal of Scientific and Research Publications, Volume 3, Issue 5, May 20131 ISSN 2250-3153.

17. Y. Filali; M. A. Sabri; A. Aarab. An improved approach for skin lesion analysis based on multiscale decomposition. 2017 International Conference on Electrical and Information Technologies (ICEIT). ISBN: 978-1-5386-1516-4. DOI: 10.1109/EITech.2017.8255250. 15-18 Nov. 2017. Rabat, Morocco.

18. Z. Waheed, An Efficient Machine Learning Approach for the Detection of Melanoma using Dermoscopic Images, pp. 316C319, 2017.

19. Y. Filali; A. Ennouni; M. A. Sabri; A. Aarab. A study of lesion skin segmentation, features selection and classification approaches. 2018 International Conference on Intelligent Systems and Computer Vision (ISCV). Pages: 1-4. ISBN: 978-1-5386-4396-9. DOI: 10.1109/ISACV.2018.8354069. 2-4 April 2018. Fez, Morocco

20. M. E. Celebi, H. A. Kingravi, B. Uddin, H. Iyatomi, Y. A. Aslandogan, W. V. Stoecker, and R. H. Moss, A methodological approach to the classification of dermoscopy images Comput. Med. Imaging Graph., vol. 31, no. 6, pp. 362C373, 2007.

21. L. I. Rudin, S. Osher, and E. Fatemi, Nonlinear total variation based noise removal algorithms, Phys. D Nonlinear Phenom., vol 60, no. 1C4, pp. 259C268, 1992.

22. J. F. Aujol, G. Aubert, L. Blanc-Fraud, and A. Chambolle, Image decomposition into a bounded variation component and an oscillating component, J. Math. Imaging Vis., vol. 22, no. 1, pp. 71C88, 2005.

23. Y. Filali, A. Ennouni, and M. A. Sabri, Multiscale approach for skin lesion analysis and classification, International Conference on Advanced Technologies for Signal and Image Processing (ATSIP). ISBN: 978-1-5386-0551-6. DOI: 10.1109/ATSIP.2017.8075545. 22-24 May 2017. Fez, Morocco.

24. R. M. Haralick, K. Shanmugam, and I. Dinstein, Textural Features for Image Classification, 1973.

25. U. T. a. Rahman, Gabor filters and gray level co occurrence matrices in texture classification, Citeseer, 2007.

26. L. Ruiz, a Fdez-Sarrła, and J. Recio, Texture feature extraction for classification of remote sensing data using wavelet decomposition: a comparative study, Int. Arch. Photogramm. Remote Sens., vol. XXXV, no. 1, pp. 1682C1750, 2004.

27. A. Marghoob, R. Braun, and A. Kopf, Interactive CD-ROM of Dermoscopy. London, U.K.: Informa Healthcare; 2007.

28. D. A. L and M. I. M, K-Means Clustering and Ensemble of Regressions: An Algorithm for the ISIC 2017 Skin Lesion Segmentation Challenge. pp. 1C5, 2017.

29. R. Amelard, S. Member, J. Glaister, S. Member, A. Wong, D. A. Clausi, and S. Member, High-Level Intuitive Features ( HLIFs ) for Intuitive Skin Lesion Description. vol. 62, no. 3, pp. 820C831, 2015.

30. A. M. Solomon, A. Murali, R. B. Sruthi, M. K. Sreekavya, S. Sasidharan, and L. Thomas, Identification of Skin Cancer based on Colour, Subregion and Texture. vol. 6, no. 7, pp. 8331C8334, 2016.

31. E. Almansour and M. A. Jaffar, Classification of Dermoscopic Skin Cancer Images Using Color and Hybrid Texture Features. vol. 16, no. 4, pp. 135C139, 2016

32. B. V Gohila and A. Selvaraj, Automated Diagnosis of Pigmented Skin Lesions Based on Texture Characteristics., vol. 3, no. 2, pp. 938C 945,2015 